Published online 2017 April 13.

Abstract

\title{
The Investigation of Lorestan University of Medical Sciences Radiology Students Attitude Toward Digital Radiology
}

\author{
Fateme Panahi, ${ }^{1, *}$ and Kourosh Gorji ${ }^{2}$ \\ ${ }^{1}$ Lorestan University of Medical Sciences, Lorestan, Iran \\ ${ }^{2}$ Babol University, Mazandaran, Iran \\ "Corresponding author: Fateme Panahi, Lorestan University of Medical Sciences, Lorestan, Iran. E-mail: panahi.fateme.radiology71@gmail.com
}

Received 2016 December 21; Accepted 2017 February 08.

\begin{abstract}
Background: Considering the graduate community in the future, defining the perception of students of radiology of the benefits of digital technology is essential to improve the quality of the images is essential. The aim of this study was to investigate students of Radiology; attitude about digital radiology in Lorestan University of Medical Sciences.

Methods: This cross-sectional study was carried out in 1393 on 38 radiology students who were selected by convenience sampling. The instrument used was a 14-item questionnaire (both digital and conventional radiology department) to assess demographic information and attitude of students of digital radiology. The validity was confirmed by experts and its reliability by Cronbakh's alpha test and SPSS19 software. At the end statistical data were analyzed using t-test and plenty of tables.

Results: The general attitude of the students was that the digital radiology, by reducing the dose can improve the density and contrast of images, enhanced details and will lead to improved radiological techniques. According to the study, 6/78 percent of students had correct information and $7.7 \%$ of them had incorrect information from digital radiology. Also the remaining respondents' information was considered as moderate.

Conclusions: In this study Radiology students believed digital radiology can enhance the production efficiency and quality of the images. They also had the right information about digital radiology. However, it is recommended in order to prevent the emergence of problems caused by change of radiology equipment and efficiency of the professional in-service training the theoretical training needs to be improved.
\end{abstract}

Keywords: Attitude, Students of Radiology, Digital Radiology

This is an abstract presented in the 33rd Iranian congress of radiology (ICR) and the 15th congress of Iranian radiographic science association (IRSA). 